\title{
ORIGINAL
}

\section{REACTIVIDAD CARDIOVASCULAR Y FACTORES DE RIESGOS CARDIOVASCULARES EN INDIVIDUOS NORMOTENSOS MENORES DE 40 AÑOS}

Mikhail Benet Rodríguez, Juan J Apollinaire Pinnini, Joel Torres Ros y Sailly Peraza Pons Facultad de Ciencias Médicas de Cienfuegos. Cuba.

\section{RESUMEN}

Fundamento: Se ha observado que la hiperreactividad cardiovascular está asociada a factores de riesgo cardiovasculares en la población general. El objetivo de este trabajo es conocer si estos factores también se asocian en la población de individuos con menos de 40 años, ya que podría ser importante para modificar estilos de vida e intervenir en la prevención de las enfermedades cardiovasculares.

Métodos: Se realizó un estudio comparativo representativo con una muestra de 249 individuos normotensos de ambos sexos entre 18 y 40 años de edad. Se formaron dos grupos a partir de los resultados de la prueba del peso sostenido (PPS), basada en la aplicación de un ejercicio isométrico para producir reactividad cardiovascular. Uno de los grupos estuvo formado por sujetos hiperreactivos cardiovasculares ( $\mathrm{n}=62$ ), con valores de presión arterial (TA) $90 / 140 \mathrm{mmHg}$ después de la prueba del peso sostenido, y el otro, estuvo formado por sujetos normorreactivos cardiovasculares $(n=187)$, con valores de TA $<90 / 140 \mathrm{mmHg}$. Mediante un modelo de regresión logística se determinó la asociación entre la edad, sexo, historia familiar de hipertensión arterial (HF de HTA), actividad física (AcF), consumo de cigarros (CC), ingestión de bebidas alcohólicas (IBA), consumo de sal (CS) y la raza con el estado de hiperreactividad cardiovascular

Resultados: El riesgo de los individuos con historia familiar de hipertensión arterial de tener hiperreactivad cardiovascular fue 2 veces mayor (OR=2,88 IC 95\%: 1,54-5,36). Por cada año de edad e riesgo de hiperreactividad aumentó en 1,08 veces y llegó hasta 1,14 veces. Por otro lado, el índice de masa corporal mostró una relación significativa independiente con el estado de hiperreactividad vascu$\operatorname{lar}(\mathrm{OR}=1,11 \mathrm{IC} 95 \%: 1,01-1,21)$. El resto de las variables estudiadas no mostraron asociación con el estado de hiperreactividad cardiovascular.

Conclusiones: Los individuos normotensos entre 18 y 40 años de edad tienen un mayor riesgo de reactividad cardiovascular si tienen historia familiar de hipertensión arterial, si su índice de masa corporal aumenta o con el aumento de la edad.

Palabras Claves: Hipertensión. Ejercicio isométrico. Enfermedades cardiovasculares. Factores de riesgo.

Correspondencia:

Dr. Mikhail Benet Rodríguez (PhD).

Departamento de Ciencias Fisiológicas. Facultad de Medicina de Cienfuegos.

Calle $51 \mathrm{~A}$ y ave 5 de septiembre. Cienfuegos, Cuba.

CP: 55100

Correo electrónico: mikhail@jagua.cfg.sld.cu

\section{ABSTRACT}

Cardiovascular Reactibity and Cardiovascular Risk Factors among Individuals under Age $\mathbf{4 0}$ with Normal Blood Pressure

Background: Cardiovascular hyperactivity has been found to be related to cardiovascular risk factors among the population as a whole. Knowing whether these factors are also related to the population of individuals under age 40 could be important for modifying lifestyles and for taking action to prevent cardiovascular diseases.

Methods: A representative comparative study was made out of 249 individuals with normal blood pressure, of both sexes, within the 18-40 age range. These individuals were divided into two groups based on the results of the hand-held weight test (HHWT), this test take into account the use of an isometric exercise to achieve cardiovascular reactivity. One of the groups was compoused of individuals with cardiovascular hyperreactivity $(\mathrm{n}=62)$, with blood pressure (BP) values of equal or higher $90 / 140 \mathrm{mmHg}$ following the handheld weight test, the other group was compoused of individuals with normal cardiovascular reactivity $(\mathrm{n}=187)$, with blood pressure values less 90/140 $\mathrm{mmHg}$. Using a logic regression model, the relationship was determined by age, sex, family history of FH of HBP), physical activity (PhysAct), cigarette-smoking (CS), alcoholic beverage intake (ABI), salt intake (SI) and race with the condition of cardiovascular hyperreactivity.

Results: The risk of having cardiovascular hyperreactivity was double for those individuals who had a family history of high blood pressure (OR=2.88 IC 95\%: $1.54-5.36)$. For every year of age, the risk of hyperreactivity increased 1.08 times, having increased up to 1.14 times. On the other hand, the body mass index revealed a significant relationship independent of the condition of vascular hyperreactivity (OR=1.11 IC 95\%: 1.01-1.21). None of the other variables studies showed any relationship to the condition of cardiovascular hyperreactivity.

Conclusions: Individuals within the 18-40 age range who have normal blood pressure, have a higher risk of cardiovascular reactivity if they have a family history of high blood pressure, if their body mass index increases and with the age increment.

Key words: Hypertension. Cardiovascular diseases. Exercice. 


\section{INTRODUCCIÓN}

La reactividad cardiovascular (RCV), es definida como los cambios en la presión de la sangre, frecuencia cardiaca u otros parámetros hemodinámicos en respuesta a un estímulo físico o mental1. La RCV exagerada o hiperreactividad cardiovascular (HRCV), como respuesta al estrés, ha sido propuesta como un factor de riesgo para la hipertensión arterial (HTA) $)^{2-4}$ y considerada como un periodo de transición entre el estado de lo que se considera tensión arterial normal al estado de HTA de un individuo determinado.

Su importancia para predecir el futuro de la hipertensión arterial y de las enfermedades cardiovasculares (ECV) está en discusión $^{1,5}$. Algunos estudios han demostrado que en la población general existe una asociación significativa entre algunos de los factores de riesgos cardiovasculares más importantes, los antecedentes familiares de hipertensión arterial y el estado de $\mathrm{HRCV}^{6}$. Además, se ha podido observar que los individuos hipertensos presentan mayor reactividad cardiovascular que los normotensos, y que sucede lo mismo en jóvenes con factores de riesgos cardiovasculares (FRCV) ${ }^{7}$.

Si se consideran todos estos antecedentes, la dificultad de crear una línea divisoria que separe la hipertensión de la tensión normal, la condición de que cuanto más baja esté la presión menor será la morbimortalidad por enfermedades cardiovasculares, y del hecho de que modificando estilos de vida poco saludables disminuye el riesgo de HTA se puede inferir la importancia que tiene estudiar la asociación entre el estado de hiperreactividad cardiovascular y los factores de riesgos cardiovasculares en individuos aparentemente normotensos con menos de 40 años de edad.

En consecuencia, este trabajo dirige la atención a determinar la asociación y el peso que tienen algunos de los FRCV más impor- tantes y el estado de respuesta cardiovascular excesiva inducida mediante el ejercicio isométrico, en personas normotensas menores de 40 años de edad.

\section{SUJETOS Y MÉTODO}

Se realizó un estudio comparativo representativo el cual tuvo como ámbito el consultorio 42 del municipio Palmira, Cuba. El universo de estudio estuvo compuesto por toda la población entre 18 y 40 años del consultorio. Se incluyó en la muestra a 249 individuos normotensos de ambos sexos. Se excluyó de la misma a las personas declaradas como hipertensas, las diagnosticadas de hipertensión arterial durante el estudio, a los portadores de cardiopatía isquémica o accidentes vasculares encefálicos y a las mujeres embarazadas. Para el diagnóstico de la situación de hipertensión se utilizó el criterio del Comité Norteamericano para el Control Evaluación y Seguimiento de la HTA ${ }^{8}$.

La determinación de los sujetos normorreactivos e hiperreactivos vasculares en los individuos normotensos se realizó mediante la "Prueba del Peso Sostenido" (PPS), una variante de las pruebas que utilizan el ejercicio físico isométrico para inducir modificaciones hemodinámicas del sistema cardiovascular $^{9}$, la cual consiste en mantener un peso de 500 gramos en la mano izquierda con el brazo extendido en ángulo recto al cuerpo durante 2 minutos. La presión arterial se toma en el brazo contrario antes del ejercicio y en los últimos 10 segundos del último minuto de éste.

Para la clasificación de los individuos en normorreactivos e hiperreactivos se utilizó el percentil 90 de las distribuciones de las presiones sistólica (PS) y/o diastólica (PD) encontradas con la PPS. Se consideró como hiperreactivas cardiovasculares (HRCV) a aquellas personas con valores iguales o superiores al 90 percentil de la PS y/o PD, y 
como normoreactivas cardiovasculares (NRCV) a las que tenían valores inferiores a este nivel. A partir de este criterio se formaron dos grupos, uno de los cuales estuvo constituido por los individuos hiperreactivos cardiovasculares, y el otro, por los individuos normorreactivos cardiovasculares.

Los factores de riesgos estudiados fueron: La edad, el sexo, la historia familiar de HTA (HF de HTA), actividad física (AcF), consumo de cigarros (CC), la ingestión de bebidas alcohólicas (IBA), el índice de masa corporal (IMC), consumo de sal (CS) y la raza.

Se consideró a un individuo como sedentario, cuando refirió no realizar ejercicio físico por lo menos 3 veces por semana durante 30 minutos o más. Los datos relacionados con la ingestión de bebidas alcohólicas fueron recogidos atendiendo al criterio de: Nunca: ingiere bebidas alcohólicas; Ocasional:1 6 veces por año; Moderado: 13 veces por semana $<100 \mathrm{ml}$.

Excesivo: 1 a 3 veces por semana > 100 $\mathrm{ml}$; Diario.

Teniendo en cuenta que $100 \mathrm{ml}$ de alcohol equivale aproximadamente a: 1/2 litro de ron; 1 litro de vino; 7 botellas de cerveza.

Esta variable se evaluó agrupando a los individuos en tres categorías: los que ingerían excesivamente o diariamente bebidas alcohólicas, los que lo hacían moderadamente y por último, los que nunca ingerían o lo hacían de manera ocasional.

Se consideró que un individuo tenía historia familiar de HTA cuando el padre, la madre o ambos eran hipertensos. En relación al consumo de cigarros se tuvo en cuenta el número de cigarros que se fumaban diariamente y se agrupó a los sujetos de la muestra en no fumadores; fumadores de menos de 10 cigarrillos diarios; entre 10 a 19 cigarrillos diarios y lo que consumían 20 o más cigarrillos diarios. Por la variable consumo de sal (CS) se agruparon como los que consumían poca sal, lo que lo hacían de manera moderada y los que consumían mucha sal.

El análisis estadístico de los datos se realizó con el programa SPSS 8. Los datos se presentan mediante números y porcentajes en tablas y gráficos, se construyó un modelo de regresión logística para rechazar la hipótesis nula de no asociación entre factores de riesgos cardiovasculares con el estado de hiperreactividad cardiovascular. Las variables independientes o predictoras de este modelo fueron: edad, IMC, sexo, HF de HTA, AcF, CS, IBA, CC y la raza.

Las variables edad e IMC tenían una distribución continua; las variables ingestión de bebidas alcohólicas, consumo de sal y hábito de fumar tenían una distribución ordinal; el resto de las variables se clasificaron como dicotómicas, donde la presencia del suceso tenia el valor 1 y la ausencia del mismo 0 . La variable dependiente en este modelo fue: respuesta cardiovascular, una variable dicotómica que tenía como posibles respuestas: hiperreactividad cardiovascular, o bien, normorreactividad cardiovascular. Los parámetros del modelo se estimaron por el método de máxima verosimilitud. Se puso como nivel de significación de inclusión de las variables predictoras el valor $\mathrm{p}<=0,05$ y de exclusión el valor $\mathrm{p}>=0,10$. Además se realizó el cálculo de la odds ratio (OR), y el intervalo de confianza del $95 \%$ (IC 95\%) para cada una de las variables predictoras que entraron en el modelo.

\section{RESULTADOS}

Los valores del percentil 90 de las distribuciones de presiones fueron de $140 \mathrm{mmHg}$ para la PS, y de $90 \mathrm{mmHg}$ para la PD. Del total de la muestra, 187 personas fueron clasificadas como normorreactivas vasculares, y 62 como hiperreactivas (figura 1).

De los 249 individuos estudiados, 111 $(44,5 \%)$ eran del sexo masculino y 138 
Figura 1

Distribución de los normorreactivos y los hiperreactivos en la muestra estudiada. (n=249). Cienfuegos, 2001

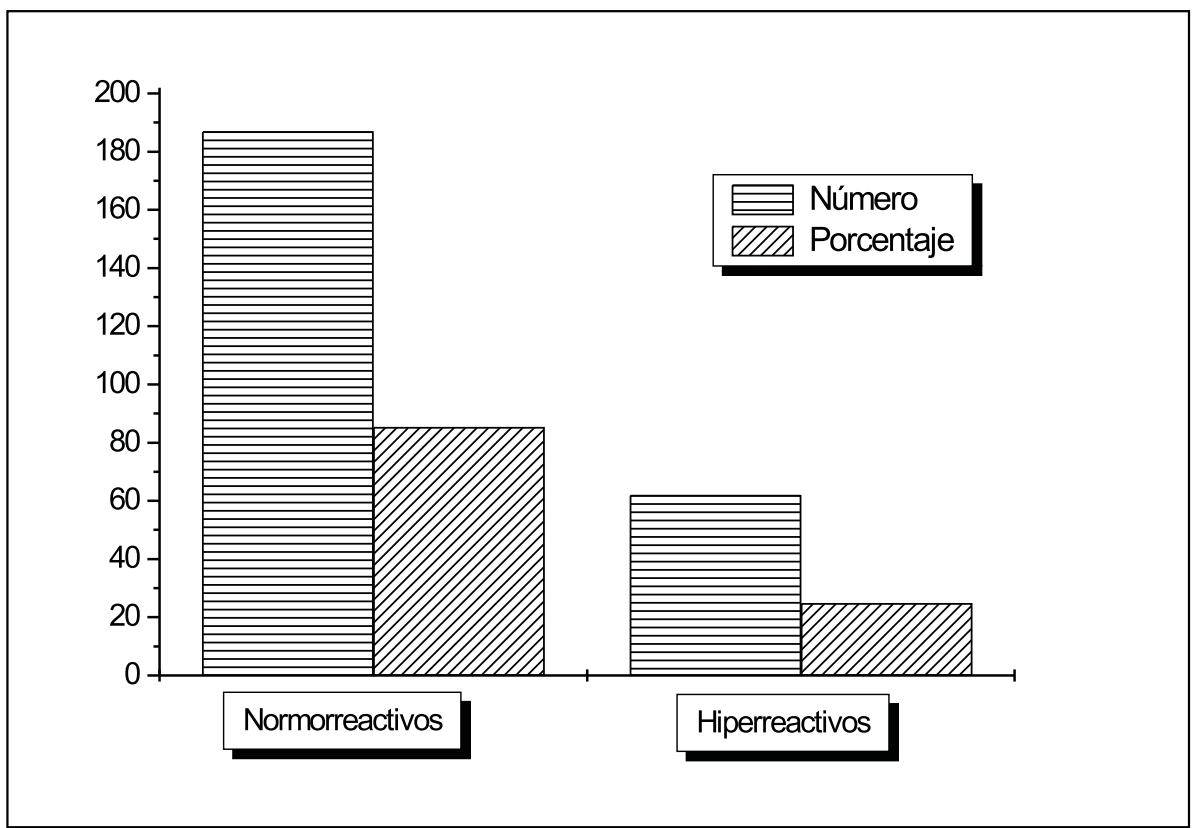

$(55,4 \%)$ del sexo femenino, la edad promedio fue de 27,8 DS 6,24 años para los normorreactivos y de 31,3 6,17 años para los hiperreactivos, y el IMC de 23,14 DS 3,03 para los primeros y de 24,71 DS 3,78 para los segundos. Las demás variables estudiadas se presentan en la tabla 1.

El riesgo de que un individuo con historia familiar de hipertensión arterial tuviese hiperreactividad vascular fue de más del doble con relación a los que no tenían estos antecedentes (OR=2,88 IC 95\%: 1,54-5,36). También resultó significativa la relación entre la edad y el riesgo de tener una hiperreactividad cardiovascular; por cada año de edad el riesgo de hiperreactividad aumentó en 1,08 veces y llegó hasta 1,14 veces. Además de estas dos variables, el IMC también mostró una relación significativa independiente con el estado de hiperreactividad vascular en las personas normotensas (OR= 1,11; IC 95\%: 1,01-1,21). El resto de las variables estudiadas no manifestaron asociación con el estado de hiperreactividad cardiovascular (tabla 2).

El índice de masa corporal fue mayor en los individuos hiperreactivos cardiovasculares que fumaban de 1 a 9 cigarros, pero disminuyó en la misma medida que aumentó el consumo de cigarros en estos individuos (figura 2).

\section{DISCUSIÓN}

Se han utilizado diferentes criterios para considerar a las personas como hiperreactivas cardiovasculares. Esto no ha sido sólo por las numerosas pruebas que se usan para llegar a este criterio, sino también por los niveles de presión que han sido considerados por diferentes autores ${ }^{4,5,9}$. El criterio que se utiliza en este estudio, sitúa los valores de presión diastólica en $90 \mathrm{mmHg}$ y de presión 
Tabla 1

Número de y porcentaje de hiperreactivos vasculares y normorreactivos vasculares en la variables consideradas en el estudio ( $n=249)$. Cienfuegos, 2001

\begin{tabular}{|c|c|c|c|c|}
\hline \multirow{2}{*}{ Variables } & \multicolumn{2}{|c|}{ Hiperreactivos } & \multirow{2}{*}{\multicolumn{2}{|c|}{$\frac{\text { Normorreactivos }}{\text { Porcentaje }}$}} \\
\hline & Número & Porcentaje & & \\
\hline \multicolumn{5}{|l|}{$\mathrm{AF}($ Sedentarismo $)$} \\
\hline $\mathrm{Si}$ & 12 & 19,4 & 64 & 34,2 \\
\hline ...No & 50 & 80,6 & 123 & 65,8 \\
\hline \multicolumn{5}{|l|}{ Sexo } \\
\hline ...Masculino & 30 & 48,4 & 81 & 43,8 \\
\hline ...Femenino & 32 & 51,6 & 106 & 56,7 \\
\hline \multicolumn{5}{|l|}{ HF de HTA } \\
\hline Con antecedentes & 34 & 54,8 & 55 & 29,4 \\
\hline ...Sin antecedentes & 28 & 45,2 & 132 & 70,6 \\
\hline \multicolumn{5}{|l|}{ Ingestión de alcohol } \\
\hline Nunca & 38 & 61,3 & 139 & 74,3 \\
\hline Modera & 17 & 27,4 & 36 & 19,3 \\
\hline Excesiva & 7 & 11,3 & 12 & 6,4 \\
\hline \multicolumn{5}{|l|}{ Consumo de sal } \\
\hline (Poca) & 10 & 16,1 & 32 & 17,1 \\
\hline ...(Moderada) & 32 & 51,6 & 111 & 59,4 \\
\hline ...(Mucha) & 20 & 32,3 & 44 & 23,5 \\
\hline \multicolumn{5}{|l|}{ Consumo de cigarros } \\
\hline ...No fuma & 40 & 64,5 & 137 & 73,3 \\
\hline ...1-9 cigarros/día & 5 & 8,1 & 6 & 3,2 \\
\hline ...10-19 cigarros/día & 6 & 9,7 & 13 & 7,0 \\
\hline ...20 o más cigarros/día & 11 & 17,7 & 31 & 16,6 \\
\hline \multicolumn{5}{|l|}{ Raza } \\
\hline Blanca & 56 & 90,3 & 163 & 87,2 \\
\hline ...Negra & 6 & 9,7 & 24 & 12,8 \\
\hline
\end{tabular}

Tabla 2

Relación factores de riesgo y estado de hiperreactividad cardiovascular (n=249). Cienfuegos, 2001

\begin{tabular}{|lccc|}
\hline Variables & OR & IC(min) & IC(max) \\
\hline Edad & $1,08^{*}$ & 1,03 & 1,14 \\
IMC & $1,11^{*}$ & 1,01 & 1,21 \\
Sexo(masculino) & 1,11 & 0,46 & 2,65 \\
HF de HTA & $2,88^{*}$ & 1,54 & 5,36 \\
AF(Sedentarismo) & 0,76 & 0,34 & 1,70 \\
IBA(moderada) & 1,54 & 0,57 & 4,09 \\
IBA(excesiva) & 1,58 & 0,39 & 6,37 \\
CS(moderada) & 1,41 & 0,50 & 4,00 \\
CS(mucha) & 1,25 & 0,49 & 3,22 \\
CC (1-9 cig/día) & 1,37 & 0,32 & 5,75 \\
CC (10-20 cig/día) & 0,64 & 0,19 & 2,10 \\
CC (+ de 20(Cig/día) & 0,55 & 0,20 & 1,51 \\
Raza (negra) & 0,93 & 0,30 & 2,90 \\
\hline
\end{tabular}

* Factores asociados con el estado hiperreactividad cardiovascular en individuos menores de 40 años 
Figura 2

Índice de masa corporal en pacientes hiperreactivos y no hiperreactivos cardiovasculares. Relación con el consumo de cigarros $(n=248)$. Cienfuegos, 2001

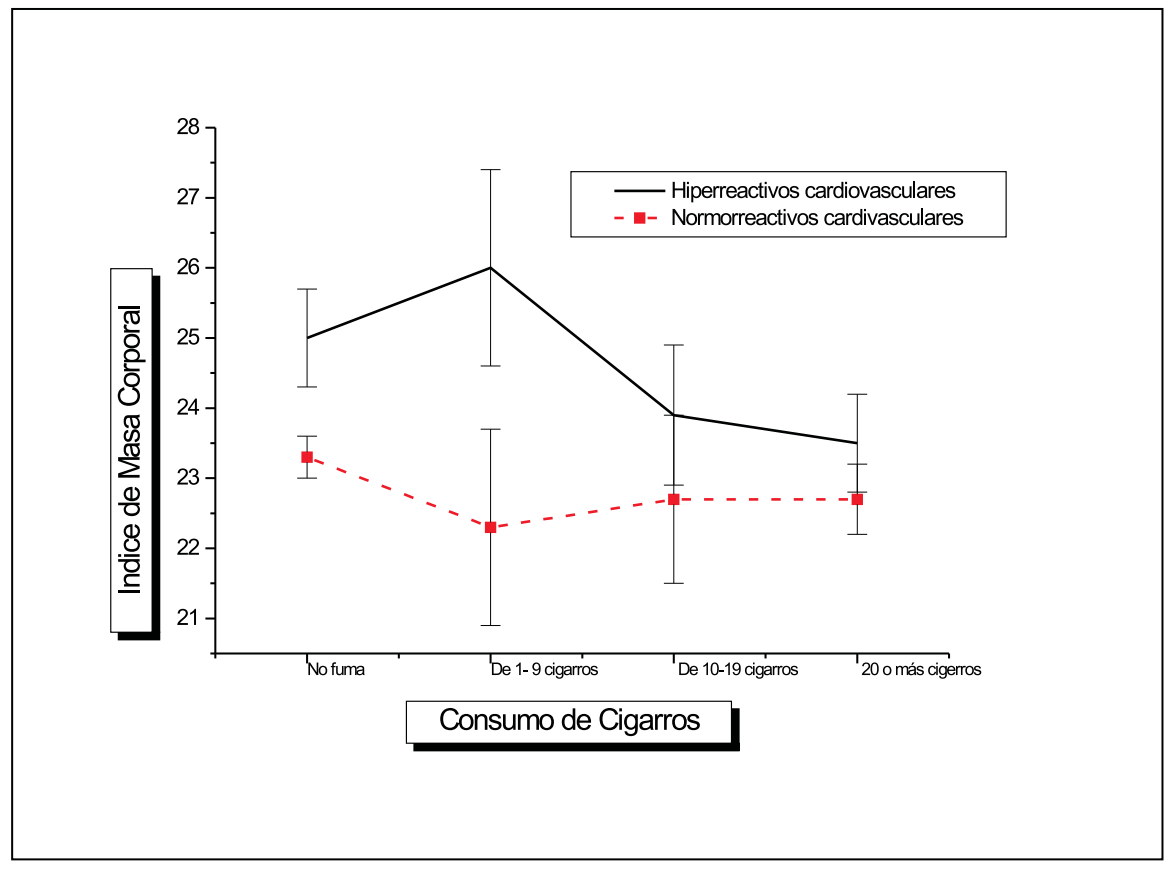

sistólica en $140 \mathrm{mmHg}$ como límites entre la normorreactividad y la hiperreactividad vascular. Este criterio disminuye este límite y por tanto aumenta el rango donde se considera a un individuo como HRCV, lo que pudiera contribuir de forma significativa en la prevención de la HTA y de las enfermedades cardiovasculares.

El principal resultado de este trabajo es que demuestra la relación que existe entre el estado de hiperreactividad cardiovascular y algunos factores de riesgos cardiovasculares (FRCV) de importancia médica, como la historia familiar de hipertensión, la edad y el IMC en individuos jóvenes normotensos. Estos resultados cobran un mayor interés si tenemos en cuenta que la hiperreactividad cardiovascular se puede considerar un factor de riesgo de la hipertensión arterial y un estado de transición desde lo que se considera normotensión a la hipertensión arterial.

Es conocido que cuando un individuo tiene historia familiar de HTA el riesgo de padecer esta entidad aumenta ${ }^{10,11}$. Se ha demostrado que los niños con historia familiar de HTA tienen niveles de presión arterial antes un estrés significativamente superiores al resto de los niños ${ }^{12}$, y que los valores de presión arterial en reposo son mayores en niños y en adolescentes con historia familiar de HTA que en los que no tienen estos antecedente13. Además, se ha observado que las personas con respuesta cardiovascular exagerada al ejercicio físico tienen un riesgo independiente de tener hipertensión arterial $^{14}$. También es conocida la relación entre la edad y el aumento del IMC con el aumento de la presión arterial ${ }^{15,16}$. Por tanto, el hecho de que este estudio muestre que 
individuos normotensos jóvenes con historia familiar de hipertensión tengan, independientemente de otros factores de riesgo, un incremento de la presión frente un estrés físico tiene una gran importancia, ya que puede ayudar a identificar a aquellos individuos con más riesgo de padecer HTA en la población, y de esa forma poder incorporar estilos de vida sanos que permitan disminuir el riesgo de esta silenciosa enfermedad.

Algunos autores plantean que la relación entre HF y HTA, parece estar dada por una acentuación de la actividad del sistema nervioso simpático, que provoca un aumento del gasto cardiaco, frecuencia cardiaca, contractilidad miocárdica y resistencia periférica total ${ }^{17,18}$. Girdler SS y otros han encontrado una mayor respuesta cardiovascular en mujeres fumadoras con respecto a los hombres fumadores ${ }^{18}$. En este trabajo no se demostró asociación significativa entre el HF y la HRCV incluso, aunque no fue significativo desde el punto de vista estadístico, se vio una disminución del riesgo de hiperreactividad en la medida que el consumo de cigarros era mayor. Es probable que estos resultados se deban a que el IMC disminuyó en los individuos hiperreactivos en la misma medida que consumían un mayor número de cigarros diarios. En otros trabajos se ha observado que los individuos que dejan de fumar tienen valores de presión más altos que los normotensos y fumadores habituales, y que el riesgo de HTA está aumentado en las personas que dejan de fumar, al parecer por el aumento del $\operatorname{IMC}^{19,20}$.

De manera contraria a la situación anterior, se observó una relación significativa entre el IMC y el riesgo de hiperreactividad cardiovascular, lo que confirma una vez más el riesgo que tienen los individuos obesos de padecer de hipertensión arterial y enfermedades cardiovasculares ${ }^{21,22}$. Además, este hallazgo apoya los resultados que demuestran que los individuos que dejan de fumar y no desarrollan acciones que impidan ganar peso, tienen un mayor riesgo de contraer hipertensión arterial.
La edad estuvo relacionada con el riesgo de hiperreactividad cardiovascular. Conocida es la asociación entre la edad y la hipertensión arterial, muchos trabajos han demostrado que la presión arterial aumenta con la edad y que ésta es un factor de riesgo independiente para las enfermedades cardiovasculares $^{23,24}$.

Aunque el sedentarismo, el consumo de alcohol y la ingestión de sal han sido asociados con la hipertensión arterial ${ }^{25,26}$, en este estudio no se demostró la relación de estas variables con el estado de hiperreactividad vascular. No obstante, es importante señalar el incremento progresivo del riesgo de hiperreactividad con el aumento del consumo de alcohol.

La lectura y discusión de estos resultados nos lleva a plantear que el riesgo de hiperreactividad cardiovascular en los individuos jóvenes normotensos se asocia significativamente con la historia familiar de HTA, el incremento de la edad y el aumento del IMC. El índice de masa corporal disminuye en las personas hiperreactivas en la medida que tienen un mayor consumo de cigarros diarios. El sexo, el consumo de sal, el consumo de alcohol y la raza negra no se asociaron de manera significativa con el estado de HRCV en estos individuos.

\section{BIBLIOGRAFÍA}

1. Falner B. The role of cardiovascular reactivity as a mediator of hypertesion in African Americans. Sem Nphrol 1996,16:117-25.

2. Wilson MF, Sung BH, Pincob GA, Lovallo WR. Exaggerated pressure responsed to exercise in men at risk for systemic hypertension. Am J Cardiol 199015,66:731-6.

3. Treiber FA, Strong WB, Arensman FW, Forrest T, Davis H, Musante L. Family history of myocardial in faretion and hemodinamic responces to exercise in young blackboys. Am J Dis Child 1991, 145:1029-33.

4. Majahalme S, Turjanmaa V, Tuomisto M, Lu H, Uusitalo A. Blood pressure responses to exercise as 
predictors of blood pressure level after 5 years. Am J Hypertens 1997,10:106-16.

5. Wennerberg SR, Schncider RH, Waloton KG, Maclean CR, Levitsky DK, Salermo JW, et al. A controlled study of the effects of the trancendental meditation program on cardiovascular reactivity and ambulatory blood pressure. Int J Neurosci 1997;89:15-28.

6. Benet M, Apollinaire JJ, González J, Yanes AJ, Fernández M. Reactividad cardiovascular y factores de riesgo cardiovascular en individuos normotensos del municipio Rodas, Cienfuegos. Rev Esp Salud Pública 1999; 73:577-584.

7. Kral BG, Becker LC, Blumenthal RS, Akersano T, Fleisher LA, Yook RM, et al Exaggerated reactivity to mental stress is associated with exercise induced myocardialischemia in an asyntomatic hig-risk population. Circulation 1997; 96:4246-53.

8. The Fifth Report of the Joint National Committee of Prevention, Detection, Evaluation and Treatment of High Blood Presure. (JNC-V). Arch Intern Med 1993;153: 154-83

9 Cantor A, Liel N. Use of isometric exercise testing can replace isotonic testing to detect and evaluate hypertensive populations. Am J Cardiol. 1990; 66:731-6.

10. Simsolo RB, Romo MM, Rabinovich L, Bonanno M, Grunfeld B. Family history of essential hypertension versus obesity as risk factors for hypertension in adolescents. Am J Hypertens 1999;12:260-

11. Burke GL, Savage PJ, Sprafka JM, Selby JV, Jacobs DR, Perkins LL, Roseman JM, Hughes GH, Fabsitz RR. Relation of risk factor levels in young adulthood to parental history of disease. The CARDIA study. Circulation 1991;84:1445-6.

12. Lemne CE. Increased blood pressure reactivity in children of borderline hypertensive fathers. J Hypertens 1998;16:1243-8.

13. Burke V, Beilin LJ, Dunbar D. Family lifestyle and parental body mass index as predictors of body mass index in Australian children: a longitudinal study. Int J Obes Relat Metab Disord 2001;25:14757.

14. Matthews CE, Pate RR, Jackson KL, Ward DS, Macera CA, Kohl HW, Blair SN. Exaggerated blood pressure response to dynamic exercise and risk of future hypertension. J Clin Epidemiol 1998; 51:29-35.
15. Sumimoto T, Hamada M, Muneta S, Shigematsu Y, Fujiwara Y, Sekiya M, Kazatani, Hiwanda K. Influence of age and severity of hipertensión on blood pressure response to isometric hangrip exesive. J Hum Hypertens 1991; 5:399-403.

16. Mayo J, Pila R, Hernández P, Pila R, Guerra C. Hipertensión arterial en el joven: factores de riesgo. Rev Med Uruguay 2000;16:24-30.

17. Winniford MD. Smoking and cardiovascular function. J Hypertens Suppl, 1990 Sep, 8:5, S17-23.

18. Girdler SS, Jamner LD, Jarvik M, Soles JR, Shapiro D. Smoking status and nicotine administration differentially modify hemodynamic stress reactivity in men and women. Psychosom Med 1997; 59:294-306

19. Halimi JM, Giraudeau B, Vol S, Caces E, Nivet H, Tichet J. Is smoking history a risk factor of arterial hypertension in men. Arch Mal Coeur Vaiss 2000; 93:949-51.

20. Lee DH, Ha MH, Kim JR, Jacobs DR. Effects of smoking cessation on changes in blood pressure and incidence of hypertension: a 4-year follow-up study. Hypertension 2001;37:194-8.

21. Ascherio A, Hennekens C, Willett WC, Sacks F, Rosner B, Manson J, Witteman J, Stampfer MJ. Prospective study of nutritional factors, blood pressure, and hypertension among US women. Hypertension 1996; 27:1065-72.

22. Jones DW. Body weight and blood pressure. Effects of weight reduction on hypertension. Am J Hypertens 1996; 9:50s-54s.

23. Sumimoto T, Hamada M, Muneta S, Shigematsu Y, Fujiwara Y, Sekiya M, Kazatani Y, Hiwada K. Influence of age and severity of hypertension on blood pressure response to isometric handgrip exercise. J Hum Hypertens 1991; 5 :399-403.

24. Tulio S, Eglé S, Greily B. Blood pressure response to exercise of obese and lean hypertensive and normotensive male adolescents. J Hum Hypertens, 1995; 9: 953-8.

25. Sircar AR, Beegom R, Singh RB, Lucknow I. Is Saturated Fat Intake A Risk Factor Of Central Obesity And Hypertension. The Canadian Journal of Cardiology supplement b June 1997; (13): 0853 .

26. Pereira MA. Physical Activity And Incidence Of Hypertension In Middle- Aged Adults. Can J Cardiol 1997; 13: A-9. 